\title{
Urinary Bladder Perforation in an Old Male with Hemorrhagic Cystitis: A Case Report
}

\author{
Nadeem Iqbal $^{{ }^{*}}$, Muhammad Haroon Khilan ${ }^{2}$, Shahzeb Irfan Malik ${ }^{3}$, Aizaz Khan ${ }^{4}$, Amna \\ Pirzada $^{5}$, Maham Khalid ${ }^{6}$, Saeed Akhter ${ }^{7}$ \\ ${ }^{1}$ Department of Urology and Kidney Transplant, Pakistan Kidney and Liver Institute \\ ${ }^{2,3,4}$ Urology Shifa college of medicine, Islamabad \\ ${ }^{5,6,7}$ Department of Urology and Kidney Transplant, Pakistan Kidney Institute, Shifa International Hospital, \\ Islamabad
}

*Corresponding Author: Nadeem Iqbal, Department of Urology and Kidney Transplant, Pakistan Kidney and Liver Institute, E-mail: dr_nadeemiqbal84@yahoo.com

\begin{abstract}
One of rare but also very severe and debilitating long-term side effect of cancer therapy is finding of hemorrhagic cystitis (HC).Radiotherapy is commonly used treatment modality for managing pelvic malignancies such as bladder, rectal, prostate and cervical cancers. Radiation cystitis is well established but rare complication. These complications may occur after few to many months of radiation therapy Here we present a case of 75 years old patient who presented to us with hemorrhagic cystitis and then due to catheter wash for bladder clots resulted in intra peritoneal bladder rupture.
\end{abstract}

\section{INTRODUCTION}

As time is passing, cancer treatment is also improving day by day. Resultantl y, there is increase in cancer patient's survival rates. Hence, cancer survivorship medical and social issues are also coming to the limelight. It's pertinent to mention here that one of rare but also very severe and debilitating long-term side effect of cancer therapy is finding of hemorrhagic cystitis (HC). Gorczynska et al [1] defined it as the manifestation of sustained hematuria accompanied with lower urinary tract symptoms in the absence of any active tumor and other conditions, such as general bleeding disorders, urinary tract infections (bacterial or fungal) and vaginal bleeding. Other than surgical modality, radiation and chemotherapy are the mostly utilized for treating cancer treatments $[2,3]$. However both of these are risk factors for the development of $\mathrm{HC}$ later on. Radiation therapy delivers high-energy rays to the tumor having less systemic side effects. However, there still is some irradiation mediated damage to surrounding normal tissue and it's inevitable. Here we present a case of 75 years old patient who presented to us with hemorrhagic cystitis and then due to catheter wash for bladder clots resulted in intraperitoneal bladder rupture.

\section{Case Presentation}

A 75 year old male patient had history of chemo radiotherapy for sigmoid cancer. He had no symptoms of lower urinary tract for many years however he developed hematuria and was brought to emergency department in our hospital. He was assessed by urology resident and was admitted for observation. $\mathrm{He}$ was catheterized to monitor hematuria and for possible start of continuous bladder irrigation in case of clots. There was nothing significant on abdominal examination such as signs of local tenderness or distension or sluggish bowel sounds. He had pulse rate of $69 /$ minute and blood pressure of 134/87 mm $\mathrm{Hg}$. He was maintaining blood oxygen saturation around 98 $\%$ on room air and he was afebrile.

Investigative work up showed complete blood picture in normal range (platelets $=163,000 / \mu \mathrm{l}$ and hemoglobin $11.2 \mathrm{~g} / \mathrm{dl}$ ).In renal function tests urea was $43 \mathrm{mg} / \mathrm{dL}$; creatinine: $1.17 \mathrm{mg} / \mathrm{dL}$. Coagulation profile was normal (INR: 1.19) . Serum electrolytes were also in normal range (sodium=131 Eq/Potassium=3.7 $\mathrm{mEq} / \mathrm{L}$ ). Creactive protein was raised $(7.9 \mathrm{mg} / \mathrm{dl})$. Ultrasound Bladder and kidneys didn't reveal abnormal echoes and there was no hydronephrosis. Diagnosis of radiotherapy 
induced hemorrhagic cystitis was made on clinical grounds as there was no evidence of other pathologies. Urine culture was sent which was negative initially. At one point the catheter was blocked so as a routine procedure it was flushed with normal saline $50 \mathrm{ml}$ and then washed to remove the blocking clots with another $500 \mathrm{ml}$ saline. He was then started continuous bladder irrigation but in few hours there was abdominal distention and signs of peritonism were seen. He was shifted to operation theatre after doing the CT scan that showed intraperitoneal bladder perforation [figure 1-3]. Peritoneal lavage and bladder repair were done.He was put on bladder irrigation with alum slowly after surgery that resolved his hematuria.

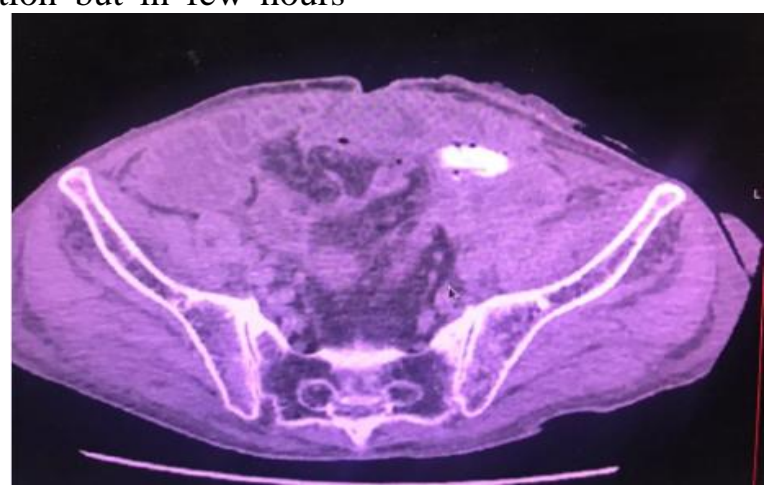

Figure1. Intraperitoneal contrast extravasation after bladder rupture

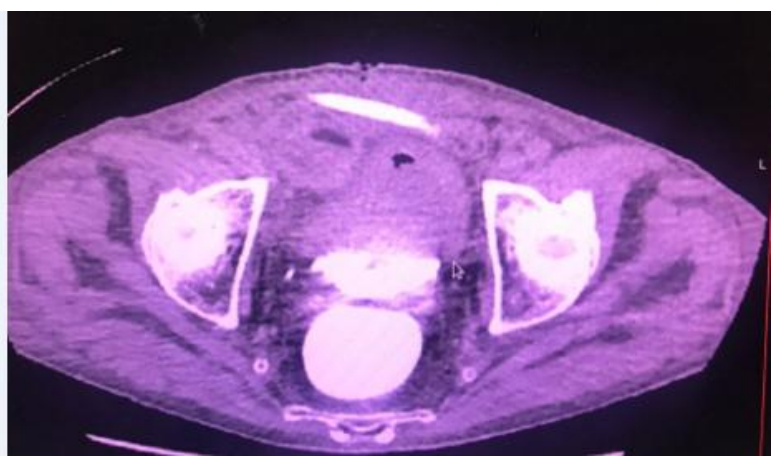

Figure2. Intraperitoneal contrast extravasation seen

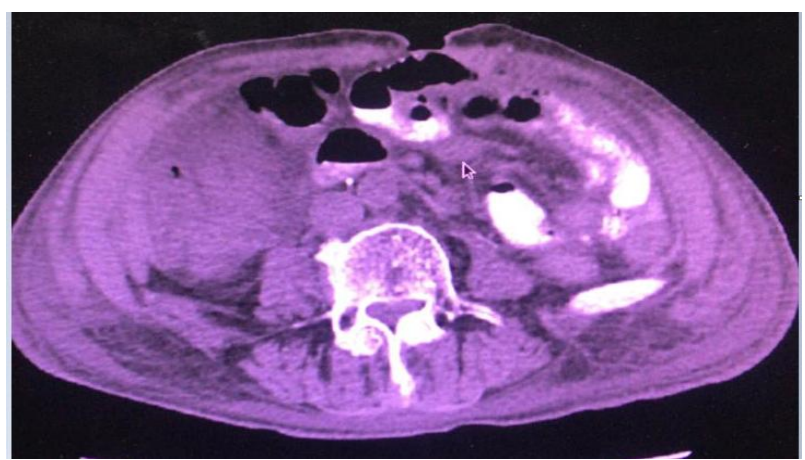

Figure3. Intraperitoneal contrast extravasation after bladder rupture

\section{DISCUSSION}

Radiotherapy is commonly used treatment modality for managing pelvic malignancies such as bladder, rectal, prostate and cervical cancers. Radiation cystitis is well established but rare complication. These complications may occur after few to many months of radiation therapy [2]. Adverse events that ae seen in HC include, dysuria, urgency, frequency, nocturia, pain in supra pubic area, fatigue, microscopic and gross hematuria. Bleeding seen in $\mathrm{HC}$ may range from nonvisible (microscopic) hematuria to gross hematuria andclots [3].

The pathophysiological mechanisms of radiation cystitisis not a well understood phenomenon. Multiple mechanisms of radiation mediated damage have been proposed till now. One of the mechanism is the inflammatory effects incurred by radiation therapy that results in trauma to the vasculature, detrusor muscle and the bladder urothelium [4]. Effects on the urothelium are evident after four months. There is cellular 
edema and nuclear irregularity, the tight junctions in urothelium and the polysaccharide layer are damaged,resulting in hypertonic urine and isotonic tissue to be in contact with one another. Late bladder fibrosis results from the damage to the vascular endothelial cells afterradiation therapy.

Complications may be associated with the indwelling urethral catheter in form of bladder stones, bleeding, urinary tract infection, and iatrogenic hypospadias [5]. Among these the drastic complication is bladderperforation that is very rare but can also be life threatening, similar to other traumatic and iatrogenic injuries [6,7].In our case as there was hematuria and patient was catheterized after that. At one point the catheter was blocked so as a routine procedure it was flushed with normal saline 50 $\mathrm{ml}$ and then washed to remove the blocking clots with another $500 \mathrm{ml}$ saline. He was then started continuous bladder irrigation but in few hours there was abdominal distention and signs of peritonism was seen. He was shifted to operation theatre after doing the CT scan that showed intraperitoneal bladder perforation. Peritoneal lavage and bladder repair were done. Intra-peritoneal bladder rupture accounts for up to $25 \%$ of cases while extra-peritoneal or a mix of the two comprises of $75 \%$ of cases. Intraperitoneal rupture mandates surgical repair [7$10]$.

In this case, patient developed intra-peritoneal bladder perforation secondary to hemorrhagic cystitis, clot formation, and then washing the clots after blockade of catheter. This underscores the fact that patient with an old history of radiotherapy and chemotherapy for pelvic organs malignancies should be dealt with extra care while managing hematuria and bladder washing for clots evacuation. They are at higher risk for bladder rupture. Bladder rupture is very rare without trauma; however it's still possible after hemorrhagic cystitis and bladder wash out for clots evacuation.

\section{REFERENCES}

[1] Gorczynska E, Turkiewicz D, Rybka K, et al. Incidence, clinical outcome, and management of virus-induced hemorrhagic cystitis in children and adolescents after allogeneic hematopoietic cell transplantation. Biol Blood Marrow Transplant. 2005; 11:797-804.

[2] S. Sutani, T. Ohashi, M. Sakayori et al., "Comparison of genitourinary and gastrointestin al toxicity among four radiotherapy modalities for prostate cancer: conventional radiotherapy, intensity-modulated radiotherapy, and permanent iodine-125 implantation with or without external beam radiotherapy," Radiotherapy and Oncology, vol. 117, no.2, pp. 27 0-276, 2015.

[3] Decker DB, Karam JA, Wilcox DT. Pediatric hemorrhagic cystitis. J Pediatr Urol. 2009;5:254264

[4] M. Rapariz-Gonz'alez, D. Castro-D'iaz, and D. Mej'ra-Rend'on, "Evaluation of the impact of the urinary symptoms on quality of life of patients with painful bladder syndrome/ chronicpelvic pain and radiation cystitis: EURCIS study," Actas Urologicas Espanolas, vol.38, no.4, pp.224-231, 2014.

[5] Iqbal N, Shah ARM, Saif UB, Hasan A, Iqbal MW, Ali M. Emphysematous Cystitis in a NonDiabetic Male. J Coll Physicians SurgPak. 2018; 28:496-497. doi:10.29271/jcpsp.2018. 06.496.

[6] O. Limon, E. E. Unluer, F. C. Unay, O. Oyar, and A. Sener, "An unusual cause of death: spontaneous urinary bladder perforation. The American journal of emergency medicine 30, no.9, pp.3-5, 2012.

[7] Stojadinovi Miroslav M., MitroviSlobodankaLj, Milovanovi Dragan R. Micropapillary carcinoma of the bladder presented with spontaneous intraperitoneal bladder rupture. Can UrolAssoc J. 2012;6(2):E42-E45. PMC. Web. 2 Dec. 2017.

[8] I. Y. Kim, S. B. Lee, B. K. Choi et al., "Bladder rupture in immediate postrenal transplant period of uncertain cause," Experimental and Clinical Transplantation, vol. 10, no. 2, pp. 180 $-182,2012$.

[9] Iqbal N, Hasan A, Malik HA, Choudhry AM, Akhter S, Nadeem U, Khan A. "Urinary Bladder Perforation Due to Urethral Catheter in an Old Lady: A Case Report". EC Clinical and Medical Case Reports. 2019; 2: 78-82.

[10] T. Nakada, H. Nakada, Y. Yoshida et al., "Hyperbaric oxygen therapy for radiation cystitis in patients with prostate cancer: a longterm follow-up study,"Urologia Internationalis, vol. 89, no.2, pp.208-214, 2012.

Citation: Nadeem Iqbal, et al. Urinary Bladder Perforation in an Old Male with Hemorrhagic Cystitis: A Case Report. ARC Journal of Urology.2019; 4(1): 1-3.doi:dx.doi.org/10.20431/2456-060X.0404001.

Copyright: () 2019 Authors. This is an open-access article distributed under the terms of the Creative Commons Attribution License, which permits unrestricted use, distribution, and reproduction in any medium, provided the original author and source are credited. 